\title{
Geostrophic Adjustment of an Isolated Diapycnal Mixing Event and Its Implications for Small-Scale Lateral Dispersion
}

\author{
M.-PASCALE LeLONG \\ NorthWest Research Associates, Bellevue, Washington \\ Miles A. Sundermeyer \\ School for Marine Science and Technology, University of Massachusetts-Dartmouth, New Bedford, Massachusetts
}

(Manuscript received 4 February 2005, in final form 1 July 2005)

\begin{abstract}
In this first of two companion papers, the time-dependent relaxation of an isolated diapycnal mixing event is examined in detail by means of numerical simulations, with an emphasis on the energy budget, particle displacements, and their implications for submesoscale oceanic lateral dispersion. The adjustment and dispersion characteristics are examined as a function of the lateral extent of the event $L$ relative to the Rossby radius of deformation $R$. The strongest circulations and horizontal displacements occur in the regime $R / L \approx O(1)$. For short times, less than an inertial period, horizontal displacements are radial. Once the adjustment is completed, displacements become primarily azimuthal and continue to stir fluid over several to tens of inertial periods. The cumulative effect of many such events in terms of the effective lateral dispersion that they induce is examined in the companion paper.
\end{abstract}

\section{Introduction}

Motivation for this two-part study comes from a series of dye tracer releases conducted as part of the Coastal Mixing and Optics experiment (henceforth $\mathrm{CMO}$ ) during late summer stratification over the New England shelf. Observed dye patterns revealed, among other things, significant lateral mixing at small scales traditionally ascribed to internal waves, which could not be reconciled with either shear dispersion or dispersion by lateral intrusions (Sundermeyer 1998; Sundermeyer and Ledwell 2001; Sundermeyer et al. 2005). A similar result was found during the North Atlantic Tracer Release Experiment (NATRE) in the pycnocline of the open ocean (Ledwell et al. 1993, 1998; Sundermeyer and Price 1998). Specifically, in that case, observed dispersion on scales of 1-10 km was 40 times that predicted by internal wave shear dispersion.

Temperature and velocity microstructure measurements taken during CMO (Oakey and Greenan 2004; Sundermeyer et al. 2005) showed the presence of

Corresponding author address: Pascale Lelong, NorthWest Research Associates, P.O. Box 3027, Bellevue, WA 98009-3027.

E-mail: pascale@nwra.com patchy regions of intense mixing superimposed on a relatively quiescent background of low diapycnal diffusivities. It is speculated that these well-mixed regions represent the end state of random, localized wave breaking or shear instability events. These turbulent regions exhibited patchiness on the same spatial scales as the dye, leading to the conjecture that the small-scale dye dispersion observed during $\mathrm{CMO}$ may be attributable to the presence of submesoscale vortical motions formed by the adjustment of such patches under gravity and rotation (Sundermeyer 1998; Sundermeyer et al. 2005). Similar interpretations have been used to explain the submesoscale dye dispersion in the NATRE data (Polzin and Ferrari 2004).

The physical situation envisioned as being responsible for the creation of isolated density anomalies is as follows: a wave packet propagates in a stably stratified region, ultimately becomes unstable to either shear or convective instability (depending upon its intrinsic frequency), and breaks. The turbulence generated by the wave breaking creates a patch of well-mixed fluid, a density anomaly, out of equilibrium with the ambient stratification. Consequently, a mass adjustment to restore equilibrium takes place. The early phase of the adjustment is characterized by a gravitational slump, 
followed by the emergence of a circulation in response to the Coriolis force.

Initially, the energy associated with the density anomaly is entirely potential. During adjustment, some of the available potential energy is converted to kinetic energy and some is radiated away as transient inertiagravity waves. The final adjusted state is sometimes referred to as the geostrophic component, the balanced part of the solution, or the vortical mode field. The unbalanced component is then the remainder, that is, the transient wave field. Ageostrophic adjustment may also occur depending on the size and intensity of the patch relative to the background stratification. However, as pertains to the problem of lateral stirring (see Sundermeyer et al. 2005), the case of geostrophic adjustment is of primary interest here.

\section{Scope and outline}

The idea that localized mixed patches resulting from diapycnal mixing events can adjust to form submesoscale coherent vortices was first investigated theoretically by McWilliams (1988, henceforth JCM88). In the same fashion as Rossby $(1937,1938)$, JCM88 used conservation laws for mass, angular momentum, and potential vorticity to relate the final adjusted state to the initially prescribed mass imbalance. This approach is quite powerful since it bypasses having to solve for the temporal evolution of the flow. However, it does not provide information about the complicated transition between initial and final states.

In the present paper, we reexamine the fluid mechanics problem posed in JCM88 by solving the initial value problem numerically in three dimensions. Like JCM88, we do not attempt to model explicitly the wave instability that creates the turbulent region [e.g., see Winters and D'Asaro (1994), Bouruet-Aubertot et al. (1996), Smyth and Moum (2000), Carnevale et al. (2001), and Staquet and Sommeria (2002) and references therein for discussion of the wave-breaking problem]. Instead, we focus on the gravitational and rotational adjustment of the well-mixed patch, once turbulent motions have ceased. Of particular interest here is the stirring ability of the resulting vortices as it relates to small-scale lateral dispersion in the ocean. We show that in general, as expected, the adjustment consists of two phases: an initial slumping followed by a longer-lived geostrophically balanced flow. In the slumping phase, fluid is displaced both radially and vertically. This phase is short lived, typically lasting less than an inertial period. In the second phase, a geostrophically balanced vortex structure develops, comprising a central anticyclone sandwiched vertically between two weaker cyclones. The ensuing flow is characterized by azimuthal displacements lasting several to many tens of inertial periods and is ultimately limited only by viscous effects. Consequently, at least for small Ekman numbers, we find that the balanced flow induces significantly more lateral dispersion than the initial slumping. This result is counterintuitive inasmuch as fluid parcels undergoing strictly azimuthal displacements are confined to circular trajectories and, hence, can only effect a limited amount of dispersion. However, as we shall show, rms displacements due to azimuthal displacements by a single vortex can nonetheless be significant. As shown in the companion paper (Sundermeyer and Lelong 2005, henceforth SL), the net dispersion due to a linear superposition of many such events can thus also be substantial.

From an oceanic dispersion perspective, the adjustment of mixed patches following diapycnal mixing events is of interest because it provides a mechanism for efficient lateral stirring at small scales, that is, horizontal scales of order $0.1-1 \mathrm{~km}$ and vertical scales of 1-10 m (e.g., Sundermeyer et al. 2005; Polzin and Ferrari 2004). From an oceanic energy budget perspective, the problem is interesting in that it provides an efficient pathway for available potential energy generated by small-scale turbulence back into kinetic energy in the form of internal waves and balanced motions (e.g., Arneborg 2002).

The goal of our two-part study is to assess the efficiency of lateral mixing by submesoscale vortical motions via numerical simulations. The objective of this first paper is to provide a detailed description of the adjustment of an isolated diapycnal mixing event. Here, to simplify our analysis, we shall assume the adjustment of a single event occurs sufficiently rapidly that viscous effects can be neglected; that is, most of the simulations and related theoretical analysis discussed here are for the inviscid regime. The cumulative effect of many such mixing events is addressed in the companion paper (SL). There, the effects of viscosity are explicitly included since they play a central role in determining the effective horizontal diffusivity.

The present paper is organized as follows: The problem is posed in section 2, and a scaling analysis is given that leads to a classification of the adjustment as a function of the ratio $R / L$, where $R$ is the Rossby radius of deformation and $L$ is the half-width of the density anomaly. Section 3 describes the numerical model and the method used to create density anomalies in a manner consistent with diffusion principles. Section 4 begins with a validation of our numerical strategy of performing simulations in a dynamically similar but reduced $N / f$ regime. Next, we examine the stages of adjustment of a density anomaly and the stirring properties of a diapycnal mixing event for $R / L=1$. We then 
explore the energetics of different $R / L$ regimes. Discussion is provided in section 5 , and concluding remarks in section 6.

\section{Background}

We consider the adjustment of a single isolated lens of mixed fluid superimposed on a background of linear stratification. As noted above, the patch is presumed to be the end result of diapycnal mixing caused by internal wave breaking. However, we do not explicitly simulate the turbulence associated with the diapycnal mixing itself. The density anomaly, or mixed patch, is assumed to have zero velocity initially. We then allow it to adjust under the influence of gravity and rotation and examine the associated vertical and lateral motions, paying particular attention to horizontal displacements and their contribution to lateral stirring.

Following Sundermeyer (1998) and Sundermeyer et al. (2005), we define the total buoyancy frequency following a mixing event as

$$
N_{\text {total }}^{2}=-\frac{g}{\rho_{0}}\left(\frac{d \bar{\rho}}{d z}+\frac{\partial \rho^{\prime}}{\partial z}\right),
$$

where $\rho^{\prime}$ is the perturbation density associated with the anomaly and $\overline{\rho(z)}$ is the linear background stratification. The change in the local buoyancy frequency between the onset and cessation of mixing is then given by

$$
\Delta N^{2}=N^{2}-N_{\text {total }}^{2},
$$

where $N^{2}=-\left(g / \rho_{0}\right) d \bar{\rho} / d z$ is the squared buoyancy frequency of the background state. In the case of complete mixing, the density within the mixed patch is uniform with depth and $\Delta N^{2}=N^{2}$. In the ocean, however, we expect $\Delta N^{2} / N^{2}$ will generally be less than 1 (e.g., Smyth and Moum 2000; Alford and Pinkel 2000). For a given anomaly strength and size, we define an associated Rossby radius of deformation:

$$
R=\frac{\Delta N h}{f},
$$

where $h$ is the vertical scale over which the stratification is modified by $\Delta N$ and $f$ is the Coriolis frequency. Based on typical $h$ and $\Delta N$ estimated from mixed patches observed during $\mathrm{CMO}$, we assume a deformation radius of $250 \mathrm{~m}$ [Sundermeyer et al. (2005) estimated an $R$ of about $350 \mathrm{~m}]$.

Previous studies of geostrophic and gravitational adjustment show that the relaxation toward a balanced state includes an initial radial slumping, accompanied by upward and downward movement of fluid below and above the center of mass of the anomaly (e.g., Lelong and McWilliams, unpublished manuscript; Stegner et al. 2004). Once the balanced state is achieved, pressure is expected to satisfy the hydrostatic relation:

$$
\begin{array}{cc}
\frac{\partial p}{\partial z} & =-g \rho \\
\left(\frac{P}{h}\right) & \left(g h \frac{\partial \rho^{\prime}}{\partial z}\right),
\end{array}
$$

where quantities in parentheses below each term represent approximations to be used later in scale analysis. From this, we obtain a pressure scale based on the anomaly strength and vertical scale, $P=\rho_{0} \Delta N^{2} h^{2}$. In the horizontal, neglecting viscous forces, the final steady-state balance necessarily involves Coriolis, inertial, and pressure gradient terms. Scaling these terms in the momentum equation with a velocity scale $U$, a horizontal length scale based on the half-width of the anomaly $L$, and the pressure scale derived above, we have

$$
\begin{array}{ccc}
f u_{\theta}+\frac{u_{\theta}^{2}}{r} & =\frac{1}{\rho_{0}} \frac{\partial p}{\partial r} \\
(f U) & \left(\frac{U^{2}}{L}\right) & \left(\frac{\Delta N^{2} h^{2}}{L}\right) \\
(\mathrm{Ro}) & \left(\mathrm{Ro}^{2}\right) & (\mathrm{Bu})
\end{array}
$$

where $u_{\theta}$ denotes the azimuthal velocity, Ro $=U / f L$ is the Rossby number, and $\mathrm{Bu}=(R / L)^{2}=\left(h^{2} \Delta N^{2}\right) /$ $\left(f^{2} L^{2}\right)$ is the Burger number. Here the Rossby radius, as defined by (3), represents the scale at which stratification and rotational effects become comparable. Specifically, on scales smaller (larger) than the Rossby radius, stratification (rotation) governs the flow behavior. Thus, three distinct regimes based on the relative magnitudes of $R$ and $L$ can be identified for the final adjusted state:

1) $R / L \approx O(1)$. This implies that $\mathrm{Ro} \approx O(1)$ and $\mathrm{Ro}^{2} \approx$ $O(1)$. The right-hand side (rhs) is balanced by both Coriolis and nonlinear terms, and the velocity scales as

$$
U \approx \frac{f L}{2}+\frac{f L}{2} \sqrt{1+4(R / L)^{2}} .
$$

This is the gradient-wind regime, sometimes referred to as the nonlinear geostrophic regime.

2) $R / L \ll O(1)$ : Then $\mathrm{Ro} \ll O(1)$ and $\mathrm{Ro}^{2} \ll O(1)$. The final balance involves the Coriolis term and the pressure gradient and

$$
U \approx \frac{\Delta N^{2} h^{2}}{f L} .
$$


This is the geostrophic equilibrium regime, sometimes called the linear geostrophic regime.

3) $R / L \gg O(1)$ : Then, $\mathrm{Ro} \gg O(1)$ and $\mathrm{Ro}^{2} \gg O(1)$. This is the cyclostrophic regime characterized by a dominant balance between the nonlinear term and the pressure gradient. In this regime,

$$
U \approx \Delta N h \text {. }
$$

In the limit of very large $R / L$, the flow responds as if there were no rotation. All of the initial PE is transferred to the transient wave field and no steady circulation is set up.

It is these three regimes that we wish to examine numerically, although we will focus primarily on the case of $R / L \approx O(1)$.

\section{Numerical method and analysis}

To examine the adjustment of mixed patches, we use a pseudospectral numerical model (Winters et al. 2004) to solve the three-dimensional, Boussinesq equations on the $f$ plane, coupled with an advection/diffusion equation for density and a passive tracer:

$$
\frac{D \mathbf{u}}{D t}+f \mathbf{i}_{3} \times \mathbf{u}=-\frac{1}{\rho_{0}} \nabla P-\mathbf{i}_{3} \frac{g}{\rho_{0}} \rho+\nu_{2} \nabla^{2} \mathbf{u}+\nu_{6} \nabla^{6} \mathbf{u},
$$

$$
\begin{aligned}
& \boldsymbol{\nabla} \cdot \mathbf{u}=0, \\
& \frac{D \rho}{D t}=\kappa_{2} \nabla^{2} \rho+\kappa_{6} \nabla^{6} \rho, \quad \text { and } \\
& \frac{D C}{D t}=\kappa_{2} \nabla^{2} C+\kappa_{6} \nabla^{6} C .
\end{aligned}
$$

Here $\mathbf{u}, P, \rho$, and $C$ denote the $3 \mathrm{D}$ velocity, the pressure, density, and tracer, respectively; $\nu_{2}$ and $\kappa_{2}$ are Newtonian viscosity and diffusivity coefficients; and $\nu_{6}$ and $\kappa_{6}$ are sixth-order hyperviscosity and hyperdiffusivity, which are employed for numerical stability. Noteworthy is our use of both Newtonian and hyperviscosity/diffusivity. While the former are physically motivated, the latter are strictly numerical, affecting only the smallest scales in both the horizontal and vertical, independent of grid resolution. This is done by scaling the hyperviscosity by the maximum nondimensional wavenumber in each coordinate direction. This, combined with the wavenumber truncation method of Patterson and Orszag (1971), is used to dissipate energy and tracer variance at the smallest scales.

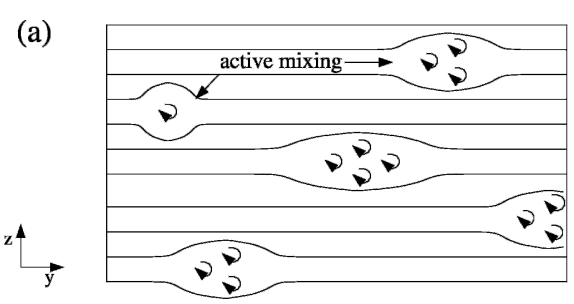

(b)

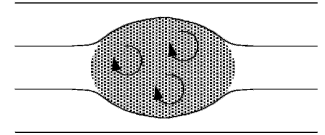

(c)

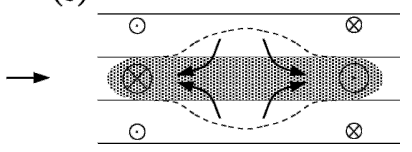

FIG. 1. Schematic of (a) modification of background stratification by random wave breaking events, (b) the well-mixed region prior to collapse, and (c) collapse inducing outward and inward radial and vertical motions, resulting in the formation of an anticyclone and two weaker cyclones. [Reprinted with permission from Sundermeyer and Ledwell (2001), copyright by the American Geophysical Union.]

\section{a. Initial conditions and model setup}

For all cases considered here, the initial condition consists of a linear background density, $\overline{\rho(z)}$, on which we superimpose a single density anomaly, intended to represent the end state of turbulent mixing associated with a localized wave breaking event. A schematic representation of anomalies of this type and their adjustment is shown in Fig. 1. Again we note our objective is not to model the dynamics of wave breaking, but rather to focus on the adjustment of the resulting weakly stratified region toward a balanced state. Details of the flow evolution prior to the formation of the well-mixed patch are not addressed.

Anomalies are introduced into the model by imposing a diffusivity $\kappa_{z}(x, y, z)$ that acts locally in space and time on the mean density profile. The form of $\kappa_{z}$ is a Gaussian in $x, y, z$; that is,

$$
\kappa_{z}(x, y, z)=\frac{1}{\Delta t} A z_{o}^{2} e^{-\left(x^{2}+y^{2}\right) /\left(2 r_{o}^{2}\right)-z^{2} /\left(2 z_{o}^{2}\right)}
$$

where $A$ is a prescribed amplitude, $r_{0}$ and $z_{0}$ are radial and vertical scales, and $\Delta t$ is the time step of the numerical diffusion calculation, which is chosen based on numerical stability constraints. Assuming an initial linear density profile in $z$, the above diffusivity yields an anomaly that is approximately $z$ Gaussian in depth and Gaussian in $x$ and $y$; that is,

$$
\rho_{\text {anomaly }}(x, y, z)=A z \frac{d \bar{\rho}}{d z} e^{-\left(x^{2}+y^{2}\right) /\left(2 r_{0}^{2}\right)-z^{2} /\left(2 z_{0}^{2}\right)} .
$$

This shape was chosen to allow comparison with the analytic solution of JCM88, that is, a two-lobed axisym- 
metric lens consisting of positive and negative density perturbations above and below the center of the anomaly. However, because we impose a diffusivity in our model rather than the actual density anomaly, there are slight differences between the actual shape of our anomaly and the analytical form given by (14). These differences, shown in Fig. 2, stem from the fact that formally (13) and (14) satisfy the finite-difference diffusion equation,

$$
\frac{\Delta \rho}{\Delta t}=\frac{\partial}{\partial z}\left(\kappa_{z} \frac{\partial \rho}{\partial z}\right)
$$

only for an initially linear density profile, $d \bar{\rho} / d z=$ const, which, for a discrete finite-difference calculation, only holds for the first time step. After that, the density gradient on the rhs is no longer constant, and the solution diverges from the single-time-step result. Put another way, for $\kappa_{z}$ given by (13), $\rho_{\text {anomaly }}$ given by (14) is not an exact solution to the continuous diffusion equation. Nevertheless, the slight differences between these solutions do not significantly affect our results. Note also that, although we integrate the above diffusion numerically, the calculations used to create the density anomalies are done "offline" in the sense that the time stepping of all other model fields is interrupted while the anomaly is being created. As a result, the anomalies are effectively generated instantaneously in the model. Last, the density anomalies used here are not sufficiently steep to develop shocks or bores at their edges such as those observed in the geostrophic adjustment of density fronts (e.g., Kuo and Polvani 1997; Stegner et al. 2004).

In all simulations, boundary conditions are triply periodic. To avoid having internal waves generated during adjustment propagate back into the domain before the vortex has equilibrated, the dimensions of our computational domain are chosen to be as large as possible relative to the wavelength of the longest radiated internal wave. Also, in all cases, we limit the time window in which we conduct our analysis to times before the fastest waves have propagated across the boundaries back into the interior of our model domain.

\section{b. N/f scaling}

The CMO dye release experiments (Sundermeyer et al. 2005) and microstructure measurements (Oakey and Greenan 2004) suggest that a large aspect ratio exists between typical horizontal $(0.1-1 \mathrm{~km})$ and vertical scales $(1-10 \mathrm{~m})$ of mixed patches. To model the adjustment of such patches, it is therefore necessary to simultaneously resolve length scales spanning several orders of magnitude, a costly endeavor in any numerical study.

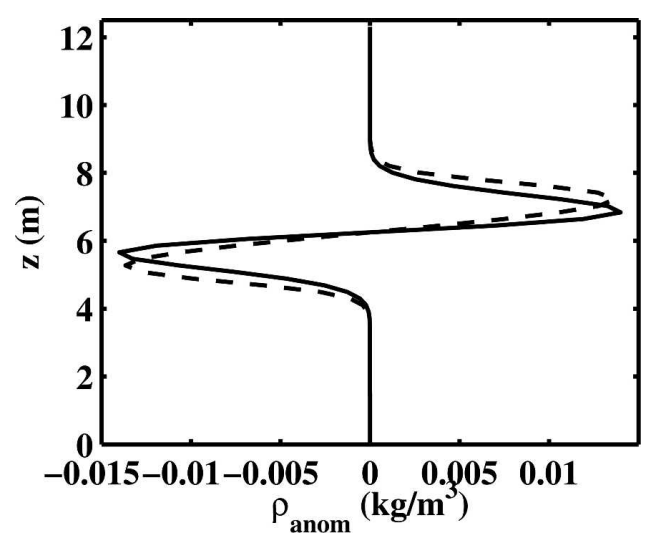

FIG. 2. Theoretical (solid) and computed (dashed) density anomalies after integration over $100 \Delta t$.

In addition, the adjustment process typically lasts a few inertial periods (the inertial period $T=12-18 \mathrm{~h}$ at midlatitudes); yet numerical stability is ensured only when the buoyancy period (on the order of minutes to tens of minutes) is well resolved. This requires a maximum time step of order tens of seconds. To avoid such high resolutions and prohibitively long integrations, the simulations presented here are performed in a regime dynamically similar to the real ocean, but numerically more tractable. This is done by varying $f$ and $L$, but keeping $N$ and $h$ fixed, so as to simultaneously increase $h / L$ and decrease $N / f$. Hence, whereas typical values of $h / L$ and $f / N$ are of order 0.01 , we use a value of 0.1 . Decreasing $N / f$ by a factor of 10 effectively reduces the Rossby radius $R$ by the same factor. Meanwhile, decreasing $L$, and hence increasing $h / L$, by the same amount maintains the relative magnitude of $L$ with respect to $R$, thereby also preserving the Burger number and the underlying dynamics. This technique has proven especially useful in the second part of our study (SL) where the model spinup from rest to a statistically equilibrated state can take hundreds of inertial periods, but where time scales of $1 / N$ must still be resolved. For such long simulations, one must additionally preserve the relative importance of viscous and diffusive forces by maintaining the Ekman number, Ek $=\nu_{2} /\left(h^{2} f\right)$. This ensures that dissipative forces will act on the same relative length and time scales in both scaled and unscaled cases. In the present single anomaly simulations, we have assumed that viscous effects are too small to affect the basic adjustment. Therefore, unless otherwise noted, simulations discussed here have been performed inviscidly. When we refer to reduced $N / f$ calculations, it will be understood that all relevant dynamical quantities, except for viscosity, have been scaled appropriately to maintain dynamical similarity. 


\section{c. Lagrangian particle and tracer statistics}

To determine net fluid displacements, and hence effective stirring during the adjustment of mixed patches, Lagrangian particles and a passive tracer were released into the model at the beginning of each run and tracked for the duration of the simulations. The initial condition for the tracer was a uniform streak in $y$ through the center of the anomaly with a Gaussian profile in $x$ and $z$. Taking advantage of the axisymmetry of the problem, floats were placed at evenly spaced radial and vertical locations in one quadrant of a vertical plane through the center of the anomaly and at regular intervals in one quadrant of a horizontal plane through the center of the anomaly.

In all cases, we report float positions relative to the center of the anomaly and displacements relative to their initial positions in either the vertical or horizontal. Relevant to the problem of lateral dispersion, we define the rms horizontal displacement of particles:

$$
D_{\mathrm{rms}}=\sqrt{\left\langle\left[x_{i}(t)--x_{i}(0)\right]^{2}+\left[y_{i}(t)--y_{i}(0)\right]^{2}\right\rangle} .
$$

The latter provides a measure of the mean displacement of fluid parcels about the anomaly, accounting for the fact that after sufficient time some of the particles will execute complete rotations about the center of the vortex. A complementary measure using the passive tracer is the horizontal tracer variance,

$$
\sigma_{x}=\frac{\iiint x C(x, y, z) d x d y d z}{\iiint C(x, y, z) d x d y d z},
$$

in the $x$ (i.e., cross streak) direction.

\section{d. Energy and energy ratios}

For any time $t$ during the adjustment process, the total energy (neglecting dissipation) can be broken down into the following component parts:

$$
\mathrm{PE}_{0}=\mathrm{PE}_{v}(t)+\mathrm{KE}_{v}(t)+\mathrm{PE}_{w}(t)+\mathrm{KE}_{w}(t),
$$

where $\mathrm{PE}_{0}$ denotes the initial potential energy associated with the density anomaly, $\operatorname{PE}_{v}(t)$ is the potential energy of the balanced flow, $\operatorname{KE}_{v}(t)$ is the kinetic energy of the balanced flow that has been converted from potential energy, and $\mathrm{PE}_{w}(t)$ and $\mathrm{KE}_{w}(t)$ are the potential and kinetic energy of the radiated waves. Actually separating wave energy from vortex energy, that is, balanced from unbalanced $\mathrm{PE}$ and $\mathrm{KE}$, in our model is not so simple, however, because wave and vortical eigenmodes do not form an orthogonal basis in a rotating system (Müller et al. 1986; Bartello 1995). Nevertheless, an approximate separation of the various energy components is possible when the adjusting flow is nonadvecting and is confined to one spatial location (as is the case for our single density anomaly on the $f$ plane). In that case, we can define the vortex energy as that contained within an inner domain centered about the initial density anomaly once all the waves have radiated away. It follows that the energy outside that domain can be attributed entirely to the wave field. Again, this method is only approximate. While outer-domain energies will always be associated with waves, for short times, innerregion energies cannot be solely attributed to the balanced component as long as waves are present. Still, as we shall show, much can be learned from this analysis.

Having distinguished the various energy components, at any time $t$, the fraction of the converted PE transferred to the wave field can be expressed as

$$
\frac{\operatorname{PE}_{w}(t)+\mathrm{KE}_{w}(t)}{\Delta \operatorname{PE}(t)}=1-\frac{\mathrm{KE}_{v}(t)}{\Delta \operatorname{PE}(t)}=1-e(t),
$$

where $\Delta \mathrm{PE}(t)=\mathrm{PE}_{0}-\mathrm{PE}_{v}(t)$. In the case of inviscid adjustment, as $t \rightarrow \infty$, the terms on the rhs of (18) asymptote to fixed values. We can thus define an energy ratio:

$$
e_{\infty}=\lim _{t \rightarrow \infty} e(t)
$$

The energy ratio $e_{\infty}$ can be interpreted as an inverse measure of wave generation (e.g., Kuo and Polvani 2000) with large (small) values indicating a weak (strong) wave field relative to balanced motions. Another good measure of the final vortex strength is the fraction of the total initial potential energy, $\mathrm{PE}_{0}$, converted to balanced $\mathrm{KE}_{v}$, that is, the ratio $\mathrm{KE}_{v} / \mathrm{PE}_{0}$. The latter is, in effect, a measure of how much of the initial potential energy is converted to balanced kinetic energy, that is, how much energy will be available for eddy stirring given a mixing event of prescribed size and strength.

\section{Results}

We now examine the adjustment process and its implications for small-scale lateral stirring, including the energetics of the three $R / L$ regimes identified in section 2. Before we proceed, however, we first verify the validity of our reduced $N / f$ scaling.

\section{a. Validation of reduced $N / f$ calculations}

The utility of performing simulations in the modified parameter regime described above was demonstrated by Lelong and Dunkerton (1998a,b) in a numerical study of inertia-gravity wave instability. To verify that 
realistic and reduced $N / f$ calculations maintain dynamical similarity for our current application, we begin by comparing the results of two simulations: one performed with a realistic value of $N / f=100$ and the other with $N / f=10$. In the reduced $N / f$ case, the horizontal extent of the anomaly relative to the Rossby radius is matched to that of the more realistic $N / f=100$ case. This ensures that both the Rossby and Burger numbers are preserved. A list of key model parameters for both simulations is given in Table 1 .

To compare the two cases, the temporal evolution of the total potential (PE) and kinetic (KE) energies, normalized by the initial PE, for both runs are shown in Fig. 3. The corresponding energies are nearly identical for the first three inertial periods and remain within a few percent of each other for the duration of the integration. This suggests that in a globally averaged sense, the two simulations behave similarly.

Plots of velocity and density taken at different times through the center of the anomaly further illustrate the similarity of the two simulations (Fig. 4). At early times, when the effects of the adjustment are limited to the near field of the anomaly, the overall structure and amplitude of the velocity and density are nearly identical. At later times the near field (now primarily associated with the balanced flow) remains similar. By contrast, the far field shows slight differences in phase and amplitude, more pronounced in the density field than in the velocity. These small differences occur because the internal wave dispersion relation does not scale exactly linearly with $N / f$. To see this, let $\omega, k$, and $m$ denote the wave frequency and horizontal and vertical wavenumbers in the $N / f=100$ case:

$$
\omega= \pm \frac{\sqrt{N^{2} k^{2}+f^{2} m^{2}}}{\sqrt{k^{2}+m^{2}}} .
$$

The corresponding quantities in the $N / f=10$ case (denoted with a subscript $r$ for "reduced") are

TABLE 1 . Model parameters for runs $N / f=10$ and $N / f=100$.

\begin{tabular}{lll}
\hline \hline Model parameters & $N / f=100$ & $N / f=10$ \\
$\begin{array}{l}\text { Domain dimensions } L_{x}, L_{y}, \\
\quad L_{z}(\mathrm{~m})\end{array}$ & $5000,5000,25$ & $500,500,25$ \\
Coriolis frequency $f\left(\mathrm{~s}^{-1}\right)$ & $9.5 \times 10^{-5}$ & $9.5 \times 10^{-4}$ \\
Buoyancy frequency $N\left(\mathrm{~s}^{-1}\right)$ & $9.5 \times 10^{-2}$ & $9.5 \times 10^{-2}$ \\
Rossby radius $R=L(\mathrm{~m})$ & 250 & 25 \\
Vertical scale $h(\mathrm{~m})$ & 1.25 & 1.25 \\
Resolution nx, ny, nz & $128,128,64$ & $128,128,64$ \\
$\Delta^{2}$ viscosity $\nu_{2}\left(\mathrm{~m}^{2} \mathrm{~s}^{-1}\right)$ & $2.5 \times 10^{-10}$ & $2.5 \times 10^{-10}$ \\
$\Delta^{2}$ diffusivity $\kappa_{2}\left(\mathrm{~m}^{2} \mathrm{~s}^{-1}\right)$ & $2.5 \times 10^{-10}$ & $2.5 \times 10^{-10}$ \\
$\Delta^{6}$ viscosity $\nu_{6}\left(\mathrm{~m}^{6} \mathrm{~s}^{-1}\right)$ & $1 \times 10^{-10}$ & $1 \times 10^{-10}$ \\
$\Delta^{6}$ diffusivity $\kappa_{6}\left(\mathrm{~m}^{6} \mathrm{~s}^{-1}\right)$ & $1 \times 10^{-10}$ & $1 \times 10^{-10}$ \\
\hline
\end{tabular}

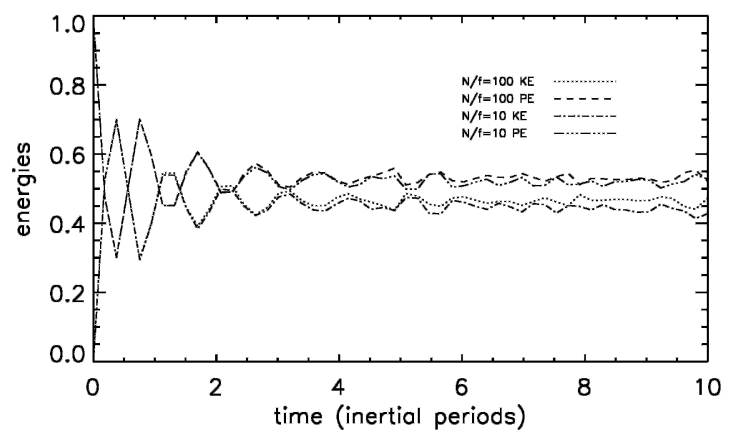

FIG. 3. Time evolution of domain-averaged energies. The inertial period corresponds to $18.4 \mathrm{~h}$ for $\mathrm{N} / \mathrm{f}=100$ and $1.84 \mathrm{~h}$ for $\mathrm{N} / f$ $=10$. All energies are normalized with the total initial PE.

$$
\omega_{r}= \pm \frac{\sqrt{N^{2} k_{r}^{2}+f_{r}^{2} m^{2}}}{\sqrt{k_{r}^{2}+m^{2}}}= \pm \frac{\sqrt{N^{2} k^{2}+f^{2} m^{2}}}{\sqrt{k^{2}+(m / 10)^{2}}} .
$$

Note that the vertical wavenumber is the same for both cases. Also, in the limit of $\mathrm{k} \ll m$, that is, for nearinertial waves, $\omega$ and $\omega_{r}$ scale linearly; in this case,

$$
\omega_{r} \approx 10 \omega .
$$

Noting that the above scaling exactly preserves the dynamics associated with geostrophically balanced/ gradient wind flow, that it approximately preserves the scaling of low-frequency internal waves (which are most important to the adjustment process since they dominate the radiated wave signal), and that any interaction between the waves (particularly the higher-frequency waves) and the geostrophic component will likely be weak (e.g., Bartello 1995), we conclude that the minor differences in the reduced $N / f$ runs are likely not important to the geostrophic component of the flow and, hence, to the evolution of the vortex structure. Indeed, we find the two simulations agree very well, suggesting that dynamical similarity is achieved as long as the relative magnitudes of all relevant parameters are preserved. Henceforth, all simulations discussed in this paper are thus performed with reduced values of $N / f$.

\section{b. Adjustment of a density anomaly with $R / L=1$}

Having verified the validity of the $N / f$ scaling, we now examine in detail the inviscid adjustment of a density anomaly with $R / L=1$. To do this, at $t=0$, a mixed patch is generated at the center of the domain and allowed to gravitationally adjust under the influence of rotation without any further external forcing. Model parameters for this run are listed in Table 2. The principal flow characteristics are presented in Fig. 5.

At very early times, the horizontal velocity field is 

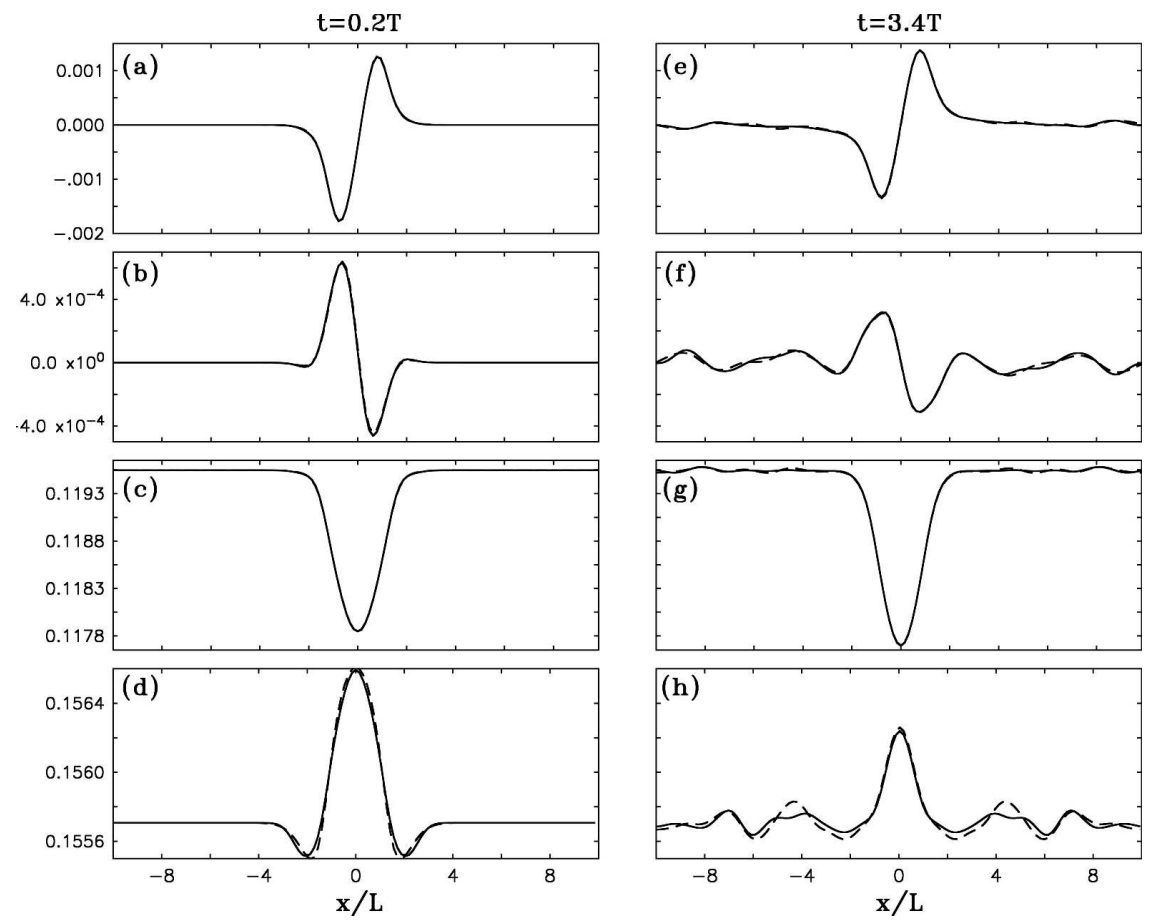

FIG. 4. Comparison of $N / f=100$ (solid) and $N / f=10$ (dashed) velocities (top four panels; units: $\mathrm{m} \mathrm{s}^{-1}$ ) and density (bottom four panels; units: $\mathrm{kg} \mathrm{m}^{-3}$ ) at $0.2 T$ and $3.4 T$ ( $T \equiv$ inertial period): (a), (e), (c), (g) at $z / h=0.64$ above the center of the anomaly (inside the anticyclonic core); (b), (f), (d), (h) at $z / h=6.4$ below the center of the anomaly (away from the core).

entirely radial (Fig. 5a), outward in the core of the anomaly and inward above and below (Fig. 5c). Very shortly thereafter, in less than an inertial period, the flow begins to respond to the Coriolis force, veering anticyclonically in the central core (Fig. 5b) and cyclonically above and below. Superimposed on these motions are pulsations in both the horizontal and vertical as waves are generated. Since there are no external forces to break the symmetry, the adjusting flow retains the radial symmetry of the initially unbalanced state. Concentric wave fronts can be seen radiating away from the anomaly core. In the vertical, a downward (upward) flow above (below) the center of the anomaly is produced in response to the early-time radial mass convergence (Fig. 5c). Once the waves have radiated away, a geostrophically balanced flow remains.

The adjusted flow consists of a three-lobed velocity distribution, a central anticyclone flanked above and below by two smaller, weaker cyclones, each comprising primarily azimuthal velocities. This three-lobed structure is particularly evident in the potential vorticity distribution (Fig. 5d). Throughout the adjustment, both density and PV anomalies retain their original functional shape but spread out laterally. The density anomaly decreases in strength, whereas PV is conserved. The adjustment is slight, however, particularly for small values of $R / L$. The anomaly undergoes several oscillations in both horizontal and vertical directions before settling down to its final state. Similar pulsations are a feature of adjusting gravity fronts (e.g., Hallworth et al. 2001; Stegner et al. 2004).

\section{c. Energy and the wave-vortex partition}

The total energy, along with inner (balanced) and outer (wave) KE and PE, are plotted in Fig. 6. Here,

TABLE 2. Model parameters for the $R / L=1$ simulation.

\begin{tabular}{lll}
\hline \multicolumn{1}{c}{ Variable } & \multicolumn{1}{c}{ Symbol } & \multicolumn{1}{c}{ Value } \\
\hline $\begin{array}{l}\text { Horizontal and vertical } \\
\text { domain size }\end{array}$ & $L_{x}, L_{y}, L_{z}$ & $\begin{array}{c}500 \mathrm{~m}, 500 \mathrm{~m}, \\
12.5 \mathrm{~m}\end{array}$ \\
Coriolis parameter & $f$ & $9.5 \times 10^{-4} \mathrm{~s}^{-1}$ \\
Background stratification & $d \bar{\rho} / d z$ & $0.037 \mathrm{~kg} \mathrm{~m}^{-4}$ \\
Anomaly amplitude & $\Delta N^{2} / N^{2}$ & 1.0 \\
Rossby radius & $R$ & $25 \mathrm{~m}$ \\
Anomaly horizontal scale & $L$ & $25 \mathrm{~m}$ \\
Anomaly vertical scale & $h$ & $1.25 \mathrm{~m}$ \\
$\nabla^{2}$ viscosity & $\nu_{2}$ & $2.5 \times 10^{-10} \mathrm{~m}^{2} \mathrm{~s}^{-1}$ \\
$\nabla^{2}$ diffusivity & $\kappa_{2}$ & $2.5 \times 10^{-10} \mathrm{~m}^{2} \mathrm{~s}^{-1}$ \\
$\nabla^{6}$ viscosity & $\nu_{6}$ & $1 \times 10^{-10} \mathrm{~m}^{6} \mathrm{~s}^{-1}$ \\
$\nabla^{6}$ diffusivity & $\kappa_{6}$ & $1 \times 10^{-10} \mathrm{~m}^{6} \mathrm{~s}^{-1}$ \\
Model time step & $d t$ & $30 \mathrm{~s}$ \\
Resolution & $\mathrm{nx}, \mathrm{ny}, \mathrm{nz}$ & $256,256,64$ \\
\hline
\end{tabular}



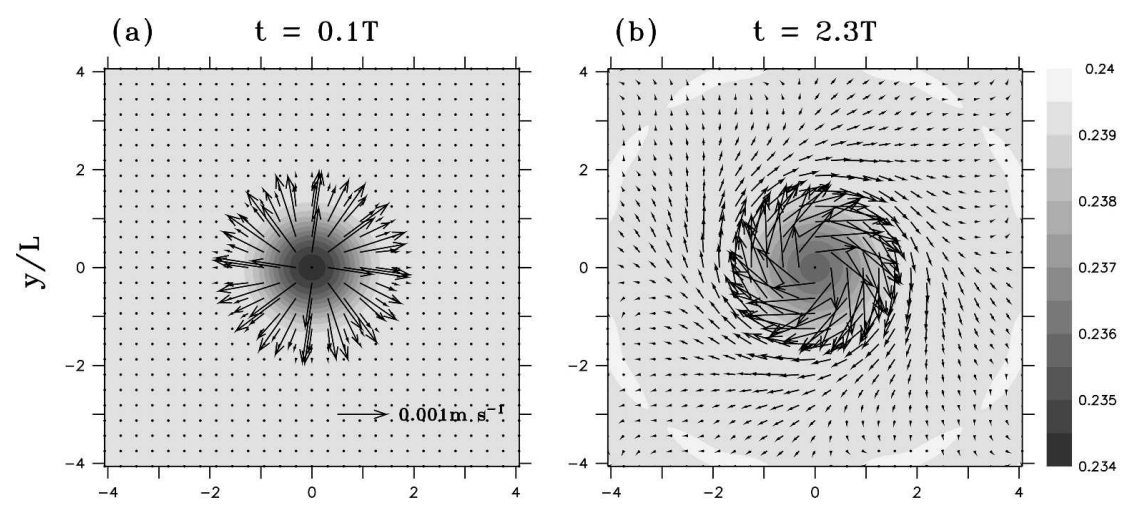

(c)

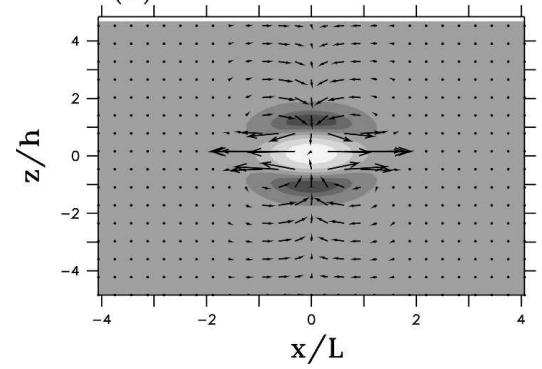

(d)

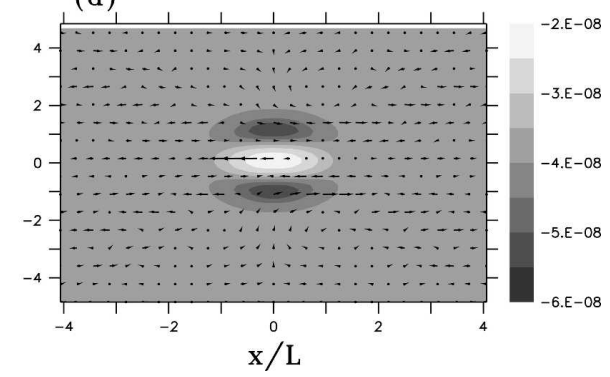

FIG. 5. Early and late time phases of adjustment ( $T \equiv$ inertial period). (a), (b) Horizontal cross sections of density $\left(\mathrm{kg} \mathrm{m}^{-3}\right)$ overlaid with velocity. Only a subregion of the total domain, centered about the initial position of the anomaly, is shown. (c), (d) Corresponding vertical cross sections of potential vorticity $\left(\mathrm{m}^{-1} \mathrm{~s}^{-1}\right)$ overlaid with velocity. Cross sections are through the horizontal plane of symmetry and slightly above the vertical plane of symmetry of the density anomaly. Top and bottom grayscales correspond to density and potential vorticity, respectively. Vector scale shown in (a) applies to all four panels.

and in what follows, we define "inner" as the region delimited by $\pm L_{x} / 8, \pm L_{y} / 8, \pm L_{z} / 8$, or 2.5 times the anomaly scale about the origin. In both regions, PE and $\mathrm{KE}$ remain out of phase with one another and continue to exchange energy periodically on an inertial time scale. Over $70 \%$ of the initial $\mathrm{PE}$ is converted to $\mathrm{KE}$ in the first one-half inertial period following collapse of the anomaly. After this initial plunge, PE relaxes back to roughly $60 \%$ of its initial value, oscillating with decreasing amplitude thereafter. Note that the equilibrium level of the inner-region energies can be deduced by temporal averaging well before the adjustment is complete. An exact time at which the adjustment is considered complete is ill defined (e.g., Kuo and Polvani 1997). However, for our problem, it is sufficient to note that much of the conversion of available PE to KE occurs rapidly, on a time scale of order one inertial period.

Additional characteristics of the wave field can be deduced from the relative sizes of wave KE and PE. For the first half-inertial period, outer-domain kinetic and potential energies grow at the same rate and are comparable in magnitude. This approximate equipartition suggests that small-scale, high-frequency waves are the first to leave the inner region. This is consistent with Gal-Chen's (1983) conclusions based on group velocity calculations that small-scale waves are the first to be generated during adjustment. Subsequently, after one to two inertial periods, the outer potential energy achieves a nearly steady state, while the outer kinetic energy continues to increase. Overall, roughly $10 \%$ of

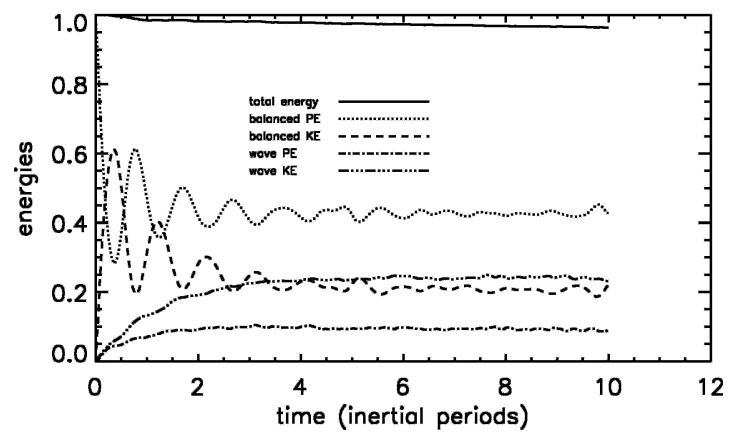

FIG. 6. Time evolution of total energy, and balanced and wave $\mathrm{KE}$ and $\mathrm{PE}$ for $R / L=1$. All energies are normalized with the total initial PE. 
the initial energy is converted to wave PE and $25 \%$ to wave KE. For later times, the frequency of the dominant wave signal may be inferred from the ratio of the wave energies. Linear inertia-gravity waves satisfy the following relation (Gill 1982):

$$
\frac{\mathrm{KE}_{w}}{\mathrm{PE}_{w}}=\frac{\omega^{2}+f^{2} \sin ^{2} \theta}{\omega^{2}-f^{2} \sin ^{2} \theta},
$$

where $\theta$ is the angle between the 3D wave vector and the horizontal plane and the wave frequency $\omega$ satisfies the dispersion relation (21). The preponderance of KE in the wave field is consistent with a low-frequency inertia-gravity wave signal, as evidenced by the observed frequency of oscillations in our model (e.g., Fig. 6).

In contrast to the wave field, most of the energy in the inner (balanced) region remains in the form of PE, with PE exceeding KE by approximately a factor of 2 . This dominance of PE is a known characteristic of balanced geostrophic flows (e.g., Gill 1982).

A time series of $e(t)$ for the $R / L=1$ case (and for additional values of $R / L$ ) is plotted in Fig. 7 (see section $4 \mathrm{e}$ for discussion of differences associated with different values of $R / L$ ). After a few large oscillations on the time scale of the inertial period, the energy ratio quickly settles toward an asymptotic value of $e_{\infty} \approx 0.35$. This value is typical of geostrophic adjustment, for which $e_{\infty}$ has been reported to lie between 0.2 and 0.5 , although $\mathrm{Ou}$ (1986) derived a theoretical value of $e_{\infty}=$ $1 / 2$ for continuously stratified geostrophic adjustment, irrespective of initial conditions.

The ratio of the balanced kinetic energy, $\mathrm{KE}_{v}$, to the initial potential energy, $\mathrm{PE}_{0}$, for different values of $R / L$ is plotted in Fig. 8. For this ratio, a maximum value of $25 \%$ is achieved for $R / L=1$. As expected, this drops quickly as $R / L$ is either increased or decreased, sup-

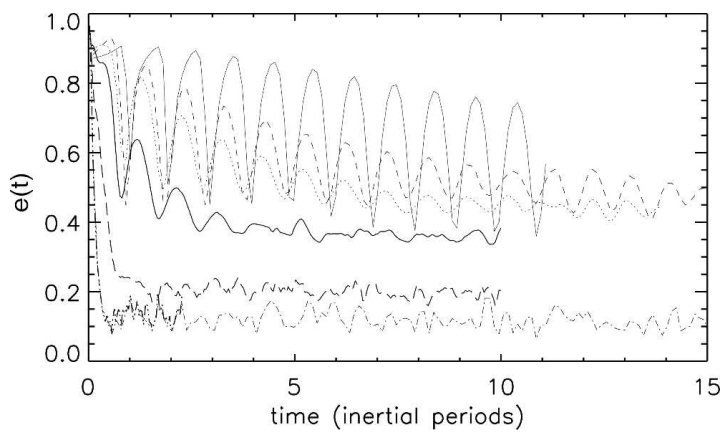

FIG. 7. Energy ratio $e(t)$ vs time for different values of $R / L$. The solid line that asymptotically approaches 0.35 corresponds to $R / L$ $=1 ; R / L$ values for the other lines as in Fig. 8 .

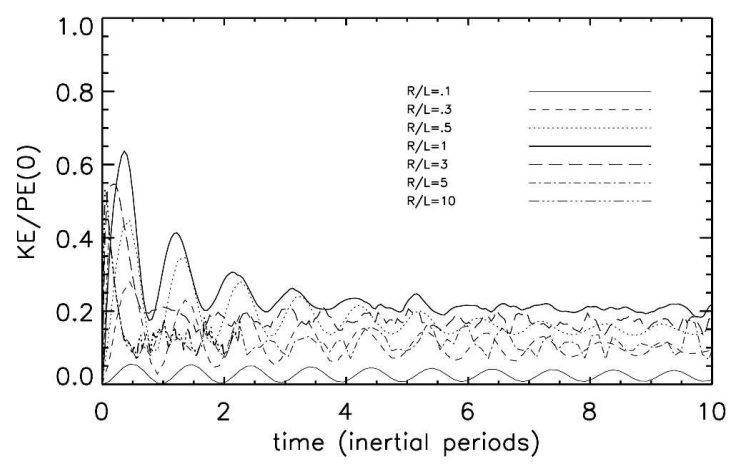

FIG. 8. Fraction of initial energy converted to balanced kinetic energy, $\mathrm{KE}_{v}$, for several values of $R / L$.

porting the idea that mixed patches of order the deformation radius will be most effective at generating balanced motions and, hence, inducing lateral dispersion.

Last, horizontal kinetic energy density spectra $|\hat{u}|^{2}$ and $|\hat{v}|^{2}$ are shown in Fig. 9. The kinetic energy is primarily concentrated at scales larger or equal to the Rossby radius and drops off at smaller scales, confirming that the adjusted state has larger radial extent than the initial anomaly. Further discussion of the spectral shape of the variance and how it relates to the multipleanomaly case can be found in SL.

\section{d. Lagrangian tracer and particle displacements}

Horizontal trajectories of floats initially positioned at regular radial intervals through the center of the anomaly and the upper cyclone are plotted in Fig. 10. Two displacement phases can be distinguished during adjustment. The first, comprising both radial and vertical displacements, is very short, lasting less than an inertial period. The second, corresponding to the more persistent, geostrophically balanced azimuthal velocities, begins once the vortex has spun up and continues for many inertial periods until eventually being dissi-

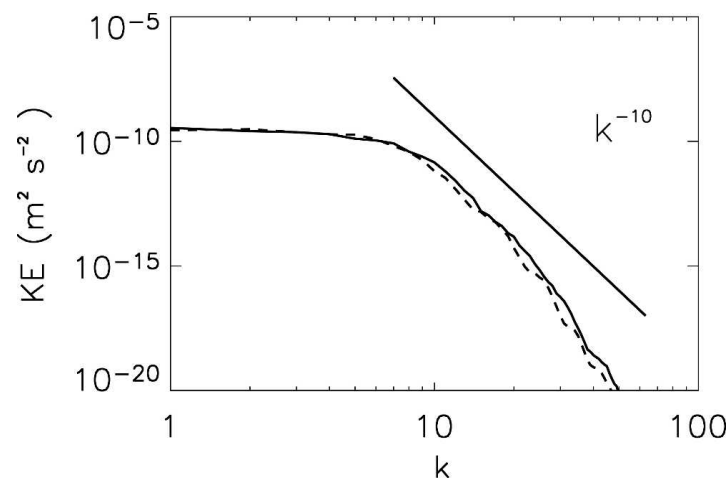

FIG. 9. Kinetic energy density spectra for $u$ (solid) and $v$ (dashed) at $t=10 T$. Rossby radius $R$, in this case is equal to $L$, corresponds to $k=20$, where $k$ is a nondimensional wavenumber. 

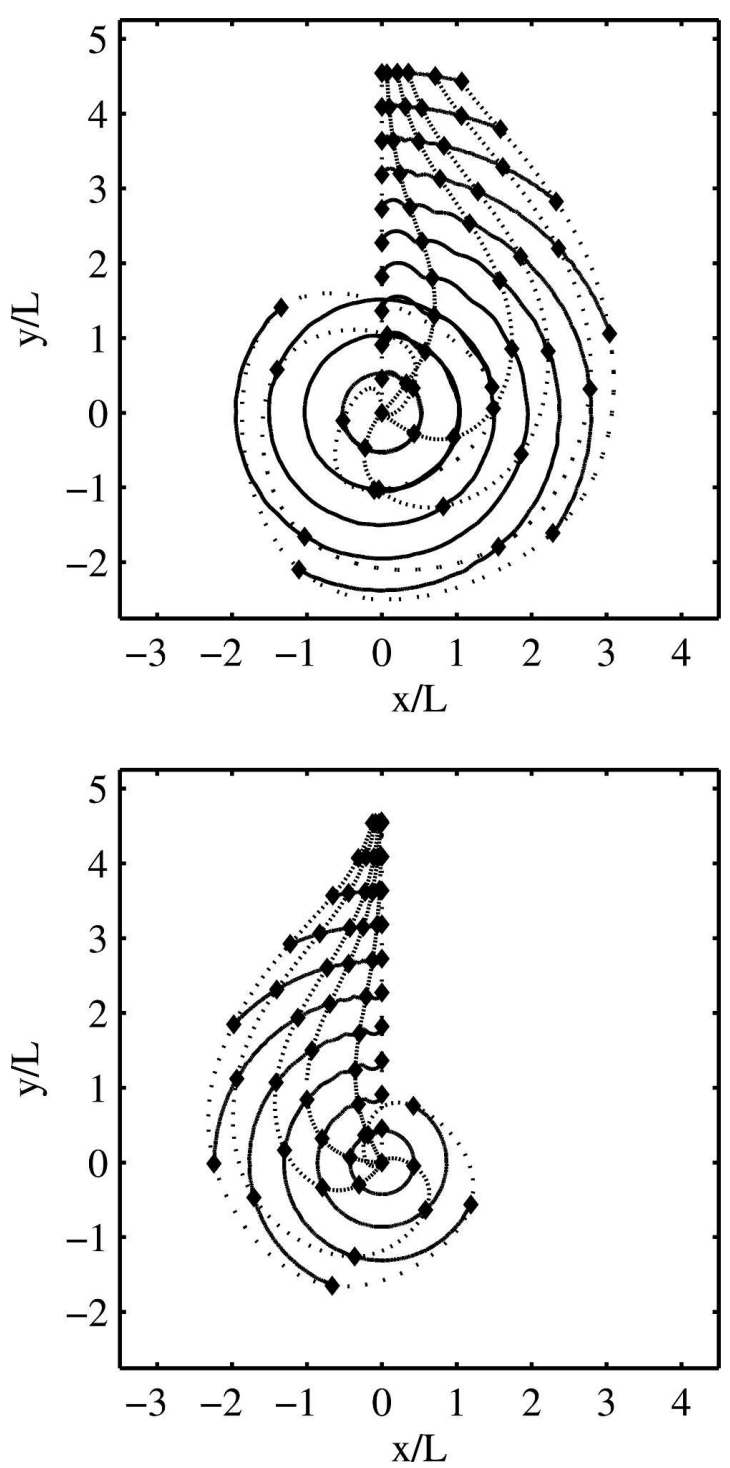

FIG. 10. Float trajectories for 10 floats positioned along $x / L=0$ $\mathrm{m}$ at $t=0$; inviscid case. (top) Float trajectories in the anticyclonic core; (bottom) floats situated above the core in the cyclone. Dots (connected by dotted lines for visual effect) denote the float positions at $t=0,1,3,5,10$, and 15 inertial periods.

pated by friction. Aside from periodic oscillations about the equilibrium position, there is no vertical displacement associated with the second phase.

Analysis of float positions 10 inertial periods into the simulation (not shown) indicates that maximum outward radial displacements occur near $r / L=0.75$ and are associated with the core of the anticyclone. Above and below the core, the formation of a weaker cyclonic circulation accompanies smaller inward radial displacements. To conserve mass, these radial motions are necessarily also accompanied by downward and upward displacements of fluid within the core of the anomaly.
This behavior agrees both qualitatively and quantitatively with the theoretical results of JCM88 (e.g., see his Figs. $3 \mathrm{f}$ and $3 \mathrm{~g}$ ).

The longer displacement phase that occurs once the vortex has spun up is dominated by azimuthal displacements. Aside from periodic oscillations about their equilibrium isopycnals, the floats do not experience any vertical or radial displacements in this phase. The largest azimuthal (i.e., angular) displacements occur for floats whose radial positions after adjustment are close to $L$ (and hence $R$ ), consistent with the fact that the azimuthal velocity reaches a maximum close to $r / R=1$. Meanwhile, particles closer to $2 L$ from the vortex center exhibit the largest rms horizontal displacements, as the time it takes them to complete a single rotation about the vortex is longer. This is evident in Fig. 11, which shows the rms displacement of a regular array of particles initially located one, two, three, four, and five deformation radii from the vortex core. The rms displacement of particles within the anomaly core reaches its maximum of just over one deformation radius after a few inertial periods, while particles situated between one and two deformation radii from the core, which have smaller rms displacements initially, tend toward larger rms displacements, up to two deformation radii, at later times. For particles much more than two radii from the core, displacements again become small as their velocities decay exponentially with increasing radius.

The time it takes a particle to execute one rotation around the core is readily estimated, given the azimuthal velocity $u_{\theta}$ and the radius of the trajectory. A

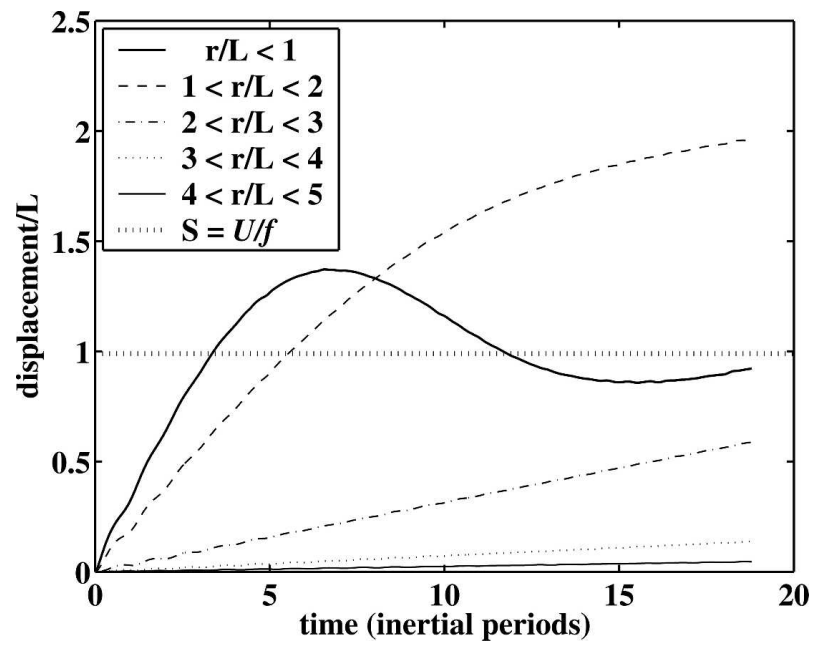

FIG. 11. Rms displacements of a regular array of particles initially placed one, two, three, four, and five deformation radii from the vortex center showing the displacement of particles well beyond a single deformation radius. Time is in inertial periods. 
particle located a distance $r$ from the anomaly center will travel $2 \pi r$ in one revolution. In this particular run, $u_{\theta}$ has a maximum value of $1.6 \times 10^{-3} \mathrm{~m} \mathrm{~s}^{-1}$ at $r=L$. It will thus take approximately nine inertial periods for fluid parcels at that location to execute a complete rotation. This is consistent with the curves plotted in Figs. 10 and 11. Immediately above and below the vortex core (e.g., Fig. 10), where the velocities are weaker, the time it takes to complete a rotation is even longer. Again, this reinforces our statement that the second phase, which we call the stirring phase, lasts significantly longer than the initial (extremely short) radial adjustment phase. Hence, it has a larger effect on lateral dispersion.

The cross-streak variance of the tracer streak shows behavior similar to $1<r / L<2$ rms particle displacements (Fig. 12; cf. with Fig. 11). Specifically, a time series of the tracer variance shows steady growth throughout the simulation, although at a slightly decreasing rate at later times. The rate of growth of the variance is many times larger than can be explained by molecular diffusion alone; that is, it is clearly the result of the advection by the vortex, not diffusion.

The above particle and tracer behaviors illustrate two important points about stirring by vortices. First, when considering all fluid parcels influenced by a vortex (i.e., not just those within the anomaly core, $r<R$ ), the rms displacement may increase for multiple eddy turnover times, not just a single eddy turnover time associated with the core of the vortex. Second, given sufficient time, the rms displacement of all particles affected by the vortex, that is, those situated more than one deformation radius from the core, may eventually grow to many times the deformation radius. This result is counterintuitive in the sense that, even though individual particles go (approximately) in circles and hence eventually return to their initial positions, the rms displacement of many such particles can still be large. This is because, despite the presence of closed streamlines, not all particles circulate about the vortex at the same rate/ frequency; hence in practice, the rms displacement of many particles never returns to zero. Instead, as shown in Fig. 11, the rms displacement initially grows monotonically and then oscillates about and eventually asymptotes to a nonzero value of order one to two deformation radii. The cumulative effect of such displacements by many vortices is the source of the lateral dispersion studied in our companion paper, SL.

\section{e. $R / L>1$ and $R / L<1$ regimes}

Up to this point we have examined the adjustment of mixed patches via the generation of vortical motions

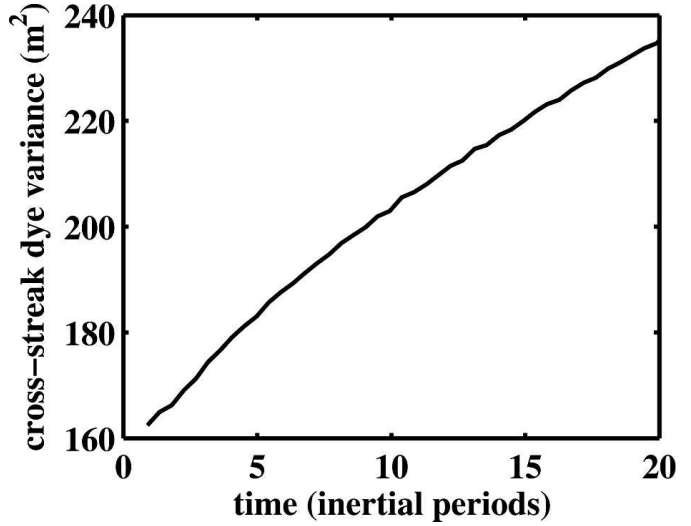

FIG. 12. Tracer variance in the $y$ (cross streak) direction as a function of time showing steady growth throughout the simulation. Time is in inertial periods.

and internal waves for the case $R / L=1$. We now briefly explore how the adjustment differs in cases where $R / L>1$ and $R / L<1$. In all cases, we hold the Rossby radius fixed at $R=25 \mathrm{~m}$ and vary the horizontal extent of the anomaly, $L$. Also, in each case, the horizontal domain size is adjusted to keep the anomaly's relative extent constant. All other parameters are unchanged from the values given in Table 2 .

The results for the $R / L>1$ cases can, in general, be interpreted in the context of the $R / L=1$ case, with a few important differences. An example using $R / L=10$ is shown in Fig. 13. First, since the initial extent of the anomaly is smaller than the Rossby radius, the anomaly initially slumps without feeling the effect of rotation. As the height of the anomaly decreases, however, the effective Rossby radius also decreases (recall that $R^{2}=$ $\Delta N^{2} h^{2} / f^{2}$ and that $\Delta N^{2}$ scales as $\left.\Delta \rho / h\right)$. Eventually the adjustment approaches the $R / L=1$ case. An important difference, however, is that by the time the radial displacement becomes comparable to the $R$ radius much of the available potential energy has already been con-

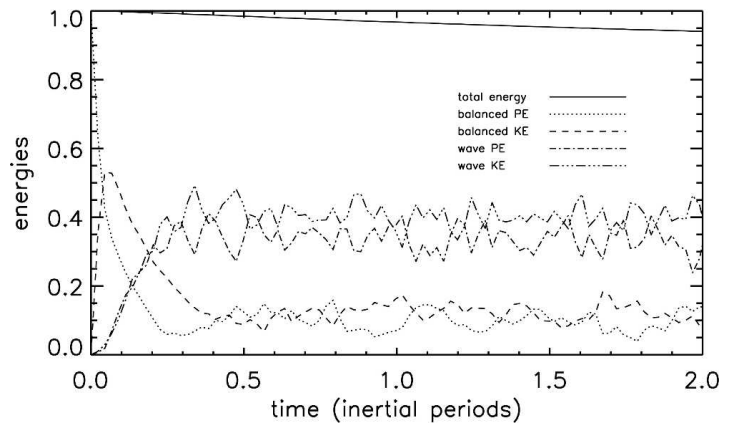

FIG. 13. Time evolution of total energy and balanced and wave $\mathrm{KE}$ and $\mathrm{PE}$ for $R / L=10$. All energies are normalized with the total initial PE (cf. with Figs. 6 and 14). 
verted to wave energy and radiated away. Consequently, the percentage of the initial potential energy available for conversion to balanced KE is small, and the resulting circulation (relative to the initial scale of the anomaly) is weak. For the case in point, the flow loses $90 \%$ of its initial PE in a quarter of an inertial period, while the inner PE is nearly an order of magnitude weaker than in the previous case (cf. with Fig. 6). Meanwhile, the outer energy is, on average, equipartitioned between $\mathrm{KE}$ and $\mathrm{PE}$, indicating that in this region the signal is dominated by high-frequency waves. The periodic exchange between KE and PE in the outer region occurs 10 times as frequently as in the previous case, that is, on the buoyancy rather than the inertial time scale. This, again, is consistent with a highfrequency wave signal. Also noteworthy in the $R / L>1$ case is that the relative size of radial displacements in comparison with azimuthal displacements (not shown) is much larger. Consequently, for early times, displacements during the initial adjustment phase are more significant than the stirring phase; that is, radial displacements dominate the dispersion.

Next, we examine an $R / L<1$ case, specifically, $R / L=0.3$ (Fig. 14). Here the radial extent of the anomaly exceeds the Rossby radius, and the flow is primarily dictated by rotational forces. In this case the flow is characterized by a very slight adjustment, confirming the well-known fact that potential energy is hard to extract from a flow dominated by rotation. Only about $25 \%$ of the initial PE is lost with less than $10 \%$ converted to KE of the balanced state. Again, the wave energies suggest that high-frequency waves are the first to radiate into the outer region, while for longer times the wave energy is primarily low frequency. Since very little of the initial available PE has been converted to $\mathrm{KE}$, the resulting vortex is again weak relative to the $R / L=1$ case. Both the $R / L>1$ and the $R / L<1$ cases thus support the idea that the most effective conversion to vortex KE, and hence the greatest lateral dispersion, will result for $R / L=1$.

Last, comparing the long-time behavior of $e(t)$ for the different cases of $R / L$, we find that in both the linear $(R / L<1)$ and nonlinear $(R / L=1)$ geostrophic cases, it asymptotes to values between 0.3 and 0.5 (see Fig. 7). In the cyclostrophic $(R / L>1)$ case (i.e., weak rotation), $e(t)$ is much smaller, indicating strong wave radiation. For all cases except the cyclostrophic regime, $e_{\infty}$ appears to be largely independent of the initial shape of the anomaly, although the time it takes for $e(t)$ to reach its asymptotic limit is much longer for small values of $R / L$. For $R / L=0.3$ and $R / L=0.1, e(t)$ undergoes large excursions that eventually converge to 0.5 . By constrast, for somewhat larger values of $R / L, e_{\infty}$ is at-

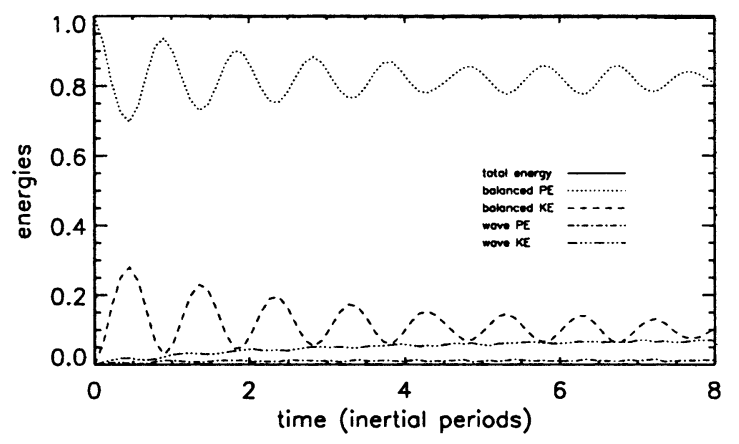

FIG. 14. Time evolution of total energy (indistinguishable from 1 in this plot) and balanced and wave $\mathrm{KE}$ and $\mathrm{PE}$ for $R / L \approx 0.3$. All energies are normalized with the total initial PE (cf. with Figs. 6 and 13).

tained within a few inertial periods. Meanwhile, the fraction of the total initial potential energy, $\mathrm{PE}_{0}$, that is converted to balanced $\mathrm{KE}_{v}$ (see Fig. 8) has a maximum value of $25 \%$ when $R / L=1$. As expected, this drops quickly as $R / L$ is either increased or decreased. Again, this supports the idea that mixed patches of the scale of the deformation radius will be most effective at generating balanced motions and, hence, inducing lateral dispersion. Somewhat surprising, however, is how readily this transition occurs as $R / L>1$ or $R / L<1$; that is, $R / L \gg 1$ or $R / L \ll 1$ is not required.

\section{f. Comparison with geostrophic scaling}

Assuming that the final density exhibits the same functional dependence as the initial anomaly (but with different eccentricity), an expression for the final velocity field follows directly from (4), (5), and (14), that is, by carrying the radial dependence of $\rho_{\text {anomaly }}$ through the hydrostatic equation to pressure, and then through the horizontal momentum equation to $u$ via $\partial P / \partial x$. In our case, the final velocity exhibits $r e^{-\alpha r^{2}}$ radial dependence where $0<\alpha<1 /\left(2 r_{0}^{2}\right)$, while its magnitude varies with $R / L$. This radial dependence agrees qualitatively with Fig. 4, which shows the azimuthal velocity as a function of distance from the core of the anomaly.

Model velocities associated with the longer-term balanced circulation can also be compared quantitatively with estimates derived from scaling arguments. Specifically, velocities of the central anticyclone predicted from the linear (6) and nonlinear (7) geostrophic expressions are plotted along with model velocities in Fig. 15 as a function of $R / L$. For all but a narrow range in which $R / L \ll 1$, model velocities are considerably smaller than scaling predictions. Two primary factors are responsible for these differences. First, as with any scaling arguments, our results only agree with the scaling to within a constant scale factor. For a Gaussian 


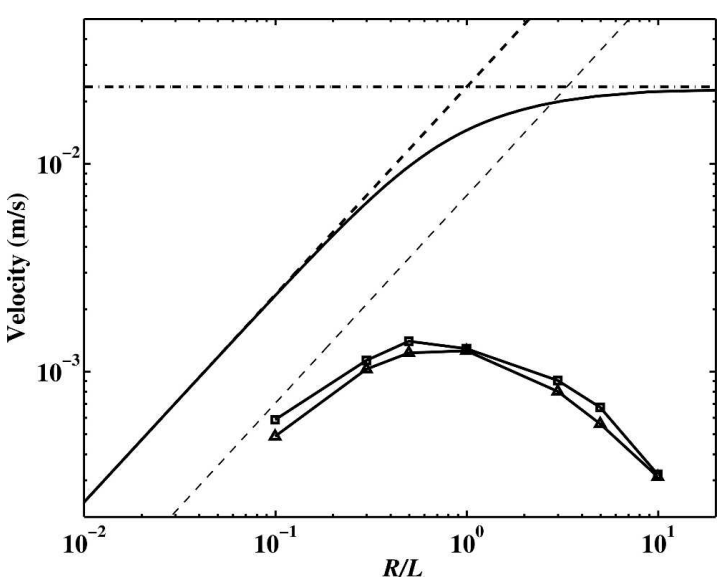

FIG. 15. Comparison of predicted velocities for geostrophic scaling (dashed), nonlinear geostrophic scaling (solid), cyclostrophic (dashed-dotted), and model-computed velocities. Triangles connected by solid lines correspond to actual model velocities, while squares connected by solid lines are based on the model pressure field, assuming geostrophic balance; i.e., $u=\left(1 / \rho_{o} f\right) \partial P / \partial x$. Theoretical scaling estimates are based on initial anomaly parameters. Thin dashed line is the geostrophic scaling result multiplied by a constant scale factor of 0.3 .

density anomaly of the form given by (14) and defining the horizontal and vertical scales as $h=2 z_{o}$ and $L=$ $2 r_{o}$, it can be shown analytically that the appropriate scale factor is $0.5 e^{-1 / 2}$, or approximately 0.3 ; that is, the maximum balanced velocity that we would expect, assuming our Gaussian anomaly is in geostrophic balance, is $0.3 \Delta N^{2} h^{2} / f L$. While it is possible to increase this scale factor to 0.6 (i.e., make it closer to order 1 ) by redefining $h$ and $L$ as $h=z_{o}$ and $L=r_{o}$, there is no analytical or computational advantage to doing so over what we have done here; hence we retain our present definitions. A second reason for the discrepancy between the predicted and actual balance velocities relates to what values we use for $\Delta N^{2}, h$, and $L$. Specifically, in the interest of clarity, we have defined these parameters (which enter into the scaling predictions for the velocities) in terms of their initial rather than final values, that is, the initial change in stratification $\Delta N^{2}$, vertical extent $h$, and horizontal extent $L$ of the mixed patches. In fact, however, over the course of adjustment, $h$ decreases, while $\Delta N^{2}$ and $L$ increase, giving an overall decrease in $U$. The degree to which the initial and final values of these parameters differ depends on $R / L$; for small $R / L$ (i.e., less than 1 ), the differences are small, while for larger $R / L$, they are larger. This is consistent with the fact that for larger $R / L$, more energy is released and radiated away as internal waves. In other words, the nature of the adjustment itself dictates the difference between the initial and balanced states. The model velocities following adjustment still agree to within a few percent of corresponding geostrophic velocities inferred from adjusted model pressure fields; that is, $u_{\text {geostr }}=\left(1 / \rho_{o} f\right) \partial P / \partial x$, provided we average over sufficient time as to remove fluctuations due to internal waves. This suggests that, although the adjusted state represents a (sometimes significantly) modified form of the initial density anomaly superimposed on any internal waves, a balanced component of the flow still exists. For large values of $R / L$, however, the contribution from internal waves can be quite large.

\section{Discussion}

\section{a. Implications to lateral dispersion}

The above energy, velocity, and displacement statistics show a number of features relevant to the problem of dispersion by the adjustment of mixed patches. First, energy ratios, $e_{\infty}$, support the notion that mixed patches with $R / L=1$ are most effective at generating lateral dispersion since, in that case, the ratio of balanced KE versus initial $\mathrm{PE}$ is largest relative to other $R / L$ values. Put another way, for a given amount of PE, that is, buoyancy production, within a mixed patch the maximal transfer to balanced KE occurs when the horizontal scale of the mixed patch is on the order of the deformation radius. Second, both rms particle displacements and tracer variance in the region of influence of the vortex grow for many, if not tens of, inertial periods and, hence, many times the classic eddy turnover time. As a result, for longer times the rms displacement of all particles influenced by the vortex can be many times the deformation radius. Furthermore, the number of particles influenced extends well beyond those within $L$ and $h$ of the anomaly core.

The latter points in particular suggest that, all else being equal, the rms lateral displacement and hence the effective lateral dispersion associated with the adjustment of mixed patches may be considerably larger than would be predicted based on the deformation radius and eddy turnover time. However, this tendency is significantly offset by the fact that the magnitude of the actual vortex velocities following adjustment are considerably smaller than those predicted from geostrophic scaling based on the initial scale of the anomaly (see Fig. 15). A major lesson we draw from this is that any number of factors could either increase or decrease the actual displacements and, hence, the dispersion generated by the adjustment of mixed patches relative to simple scaling estimates. Simulations such as the ones presented here and in SL, which include a more complete description of the relevant dynamics, are therefore necessary to evaluate these competing factors. 


\section{b. Viscous effects}

In the above analysis, we have concentrated solely on the problem of inviscid adjustment of mixed patches. Ultimately, however, the total displacement resulting from the generation and eventual decay of a vortex also depends on processes acting on very long time scales, namely dissipative forces. To underscore this point, a final simulation was run similar to our $R / L=1$ base run but with a larger, more realistic (scaled) viscosity value of $1.0 \times 10^{-5} \mathrm{~m}^{2} \mathrm{~s}^{-1}$. Resulting displacements are shown in Fig. 16. As anticipated, for the first one to two inertial periods the trajectories are indistinguishable from the inviscid case. Subsequently, however, the effects of viscous damping become evident. In both the core anticyclone and in the overlying cyclone, net azimuthal displacements are significantly smaller than in the inviscid case. Comparison of Figs. 10 and 16 also shows that viscosity damps out the smaller oscillatory excursions due to internal waves. As will be shown in $\mathrm{SL}$, this damping of azimuthal displacements can significantly reduce the overall lateral dispersion by adjusting mixed patches.

\section{Conclusions}

Numerical simulations of the time-dependent adjustment of a density anomaly have shown that, for anomalies of a given amplitude, the ones with dimensions $R / L=1$ are the most efficient generators of balanced vortical motions. In this case, the vortices produced are efficient at stirring fluid over long periods of time. By contrast, for $R / L<1$, rotational effects dominate the adjustment process and very little of the initial available potential energy is converted to kinetic energy. Consequently, for a given amount of available PE, the vortices produced are weaker and do not generate as much stirring. Last, when $R / L>1$, much of the potential energy is converted to wave energy during the initial stages of the adjustment, and the resulting vortex, if it forms at all, is again weak. It is not clear, at this point, whether the preponderance of $R / L \approx O(1)$ structures in the $\mathrm{CMO}$ and concurrent microstructure datasets means that most adjusting features tend to have dimensions in that regime. It may be that these features are the most likely to be observed precisely because they are the most robust and able to withstand dissipative effects. A careful study of the dynamics of wave breaking events would help in answering this question.

Regarding Lagrangian and particle displacements associated with the adjustment of mixed patches, we draw the following lessons. First, given sufficient time, the rms displacement of particles, as well as the variance of a passive tracer in and around the vortex, may be many
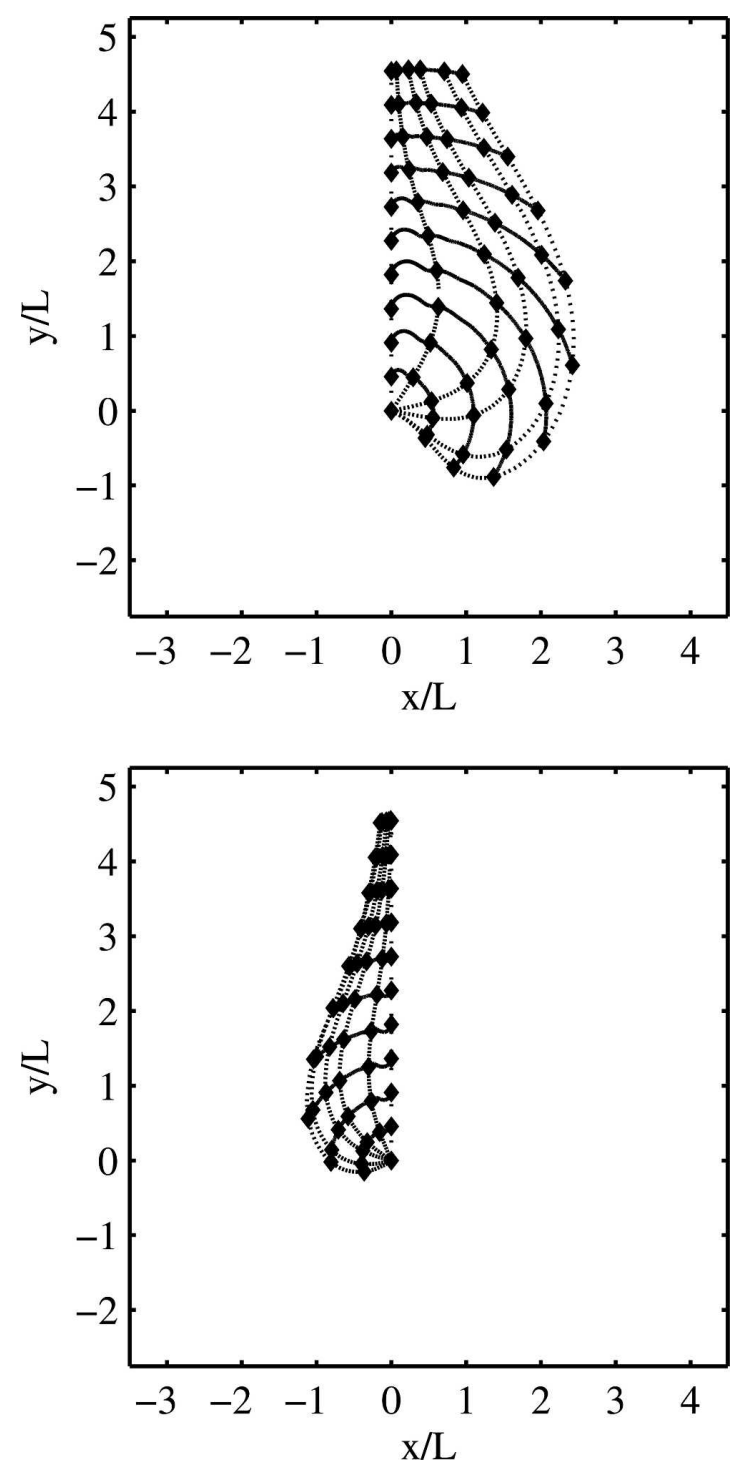

FIG. 16. Float trajectories for 10 floats positioned along $x / L=0 \mathrm{~m}$ at $t=0$; viscous case. (top) Float trajectories in the anticyclonic core; (bottom) floats situated in the cyclone above the core. Dots represent the float positions at $t=0,1,3,5,10$, and 15 inertial periods.

times the local deformation radius, $R=\Delta N h / f$, associated with the mixed patch. Second, because fluid parcels far from the anomaly core can take many, possibly even tens of, inertial periods to complete a single rotation about the vortex, such displacements may occur over time scales many times the conventional eddy turnover time. Third, balanced velocities associated with fully adjusted mixed patches may be many times smaller than predicted based on linear and/or nonlinear geostrophic scaling using the initial scales of mixed patches. The latter is in part due to an inherent scale factor and partly the result of the increase in the aspect ratio (horizontal:vertical) of mixed patches that occurs 
during the adjustment. Last, viscosity does not significantly inhibit the initial adjustment of mixed patches but tends to arrest motions associated with the balanced state, including azimuthal displacements, which are primarily responsible for lateral displacements. Thus, this limits the overall lateral dispersion by these events.

The above characteristics have significant implications to the overall dispersion by the adjustment of mixed patches following diapycnal mixing events. In the present study, we have attempted to highlight which of these are well predicted by theory and which are not. In summary, we conclude that there are numerous factors that can affect the ultimate rate at which fluid parcels are stirred by the adjustment of such events. In some cases, simple scaling arguments would underpredict the resulting dispersion, while in other cases they would overpredict it. Whether models such as the one presented here and in SL incorporate enough of the relevant dynamics to enable an accurate prediction of the actual dispersion by the adjustment of mixed patches remains to be tested in the field.

Acknowledgments. This work was funded by the Office of Naval Research under Grant N00014-01-1-0984. Author M.-P. Lelong also received support from NSFPO-9811939. The authors thank Eric Kunze, Kurt Polzin, and Raffaele Ferrari for insightful discussions; Kraig Winters for the use of his numerical model; and Bill Smyth and an anonymous reviewer for helpful comments on the manuscript.

\section{REFERENCES}

Alford, M. H., and R. Pinkel, 2000: Observations of overturning in the thermocline: The context of ocean mixing. J. Phys. Oceanogr., 30, 805-832.

Arneborg, L., 2002: Mixing efficiencies in patchy turbulence. $J$. Phys. Oceanogr., 32, 1496-1506.

Bartello, P., 1995: Geostrophic adjustment and inverse cascades in rotating stratified turbulence. J. Atmos. Sci., 52, 4410-4428.

Bouruet-Aubertot, P., J. Sommeria, and C. Staquet, 1996: Stratified turbulence produced by internal wave breaking. Dyn. Atmos. Oceans, 285, 265-301.

Carnevale, G. F., M. Briscolini, and P. Orlandi, 2001: Buoyancy-to inertial-range transition in forced stratified turbulence. $J$. Fluid Mech., 427, 205-239.

Gal-Chen, T., 1983: Initialization of mesoscale models: The possible impact on remotely sensed data. Mesoscale Meteorology: Theory, Observations and Models, D. Lilly and T. GalChen, Eds., Reidel, 157-171.

Gill, A. E., 1982: Atmosphere-Ocean Dynamics. Academic Press, $662 \mathrm{pp}$.

Hallworth, M. A., H. E. Huppert, and M. Ungarish, 2001: Axisymmetric gravity currents in a rotating system: Experimental and numerical investigations. J. Fluid Mech., 447, 1-29.

Kuo, A. C., and L. M. Polvani, 1997: Time-dependent fully nonlinear geostrophic adjustment. J. Phys. Oceanogr., 27, 1614-1634.

-, and _ 2000: Nonlinear geostrophic adjustment, cyclone/ anticyclone asymmetry, and potential vorticity rearrangement adjustment. Phys. Fluids, 12, 1087-1100.

Ledwell, J. R., A. J. Watson, and C. S. Law, 1993: Evidence for slow mixing across the pycnocline from an open ocean tracer release experiment. Nature, 364, 701-703.

,-- , and 1998: Mixing of a tracer in the pycnocline. $J$. Geophys. Res., 103, 21 499-21 529.

Lelong, M.-P., and T. J. Dunkerton, 1998a: Inertia-gravity wavebreaking in three dimensions. Part I: Convectively stable waves. J. Atmos. Sci., 55, 2473-2488.

$\_$, and -1998 b: Inertia-gravity wavebreaking in three dimensions. Part II: Convectively unstable waves. J. Atmos. Sci., 55, 2489-2501.

McWilliams, J. C., 1988: Vortex generation through balanced adjustment. J. Phys. Oceanogr., 18, 1178-1192.

Müller, P., G. Holloway, F. Henyey, and N. Pomphrey, 1986: Nonlinear interactions among internal gravity waves. Rev. Geophys., 24, 493-536.

Oakey, N. S., and B. J. W. Greenan, 2004: Mixing in a coastal environment: 2. A view from microstructure measurements. J. Geophys. Res., 109, C10014, doi:10.1029/2003JC002193.

Ou, H. W., 1986: On the energy conversion during geostrophic adjustment. J. Phys. Oceanogr., 16, 2203-2204.

Patterson, G. S., and S. A. Orszag, 1971: Spectral calculations of isotropic turbulence: Efficient removal of aliasing interactions. Phys. Fluids, 14, 2538-2541.

Polzin, K., and R. Ferrari, 2004: Isopycnal dispersion in NATRE. J. Phys. Oceanogr., 34, 247-257.

Rossby, C., 1937: On the mutual adjustment of pressure and velocity distributions in certain simple current systems, I. $J$. Mar. Res., 1, 15-28.

_ 1 1938: On the mutual adjustment of pressure and velocity distributions in certain simple current systems, II. J. Mar. Res., 1, 239-263.

Smyth, W. D., and J. N. Moum, 2000: Length scales of turbulence in stably stratified mixing layers. Phys. Fluids, 12, 1327-1342.

Staquet, C., and J. Sommeria, 2002: Internal gravity waves: From instabilities to turbulence. Annu. Rev. Fluid Mech., 34, 559-593.

Stegner, A., P. Bouruet-Aubertot, and T. Pichon, 2004: Nonlinear adjustment of density fronts. Part I: The Rossby scenario and the experimental reality. J. Fluid Mech., 502, 335-360.

Sundermeyer, M. A., 1998: Studies of lateral dispersion in the ocean. Ph.D. thesis, Massachusetts Institute of TechnologyWoods Hole Oceanographic Institution Joint Program, Woods Hole, MA, 215 pp.

_ and J. F. Price, 1998: Lateral mixing and the North Atlantic Tracer Release Experiment: Observations and numerical simulations of Lagrangian particles and a passive tracer. $J$. Geophys. Res., 103, 21 481-21 497.

_ , and J. R. Ledwell, 2001: Lateral dispersion over the continental shelf: Analysis of dye-release experiments. J. Geophys. Res., 106, 9603-9621.

_ , and M.-P. Lelong, 2005: Numerical simulations of lateral dispersion by the relaxation of diapycnal mixing events. $J$. Phys. Oceanogr., 35, 2368-2386.

_, J. R. Ledwell, N. S. Oakey, and B. J. W. Greenan, 2005: Stirring by the relaxation of diapycnal events. J. Phys. Oceanogr., 35, 1245-1262.

Winters, K. B., and E. A. D'Asaro, 1994: Three-dimensional wave instability near a critical level. J. Fluid Mech., 272, 255-284.

, J. A. MacKinnon, and B. Mills, 2004: A spectral model for process studies of rotating, density-stratified flows. J. Atmos. Oceanic Technol., 21, 69-94. 